\title{
Effect of Plastic Deformation on Magnetic Properties of Nickel and Interpretation of an Early Self-Reversal of Magnetization Observed by Nagaoka
}

\author{
R.S. CARMichael and M.D. Fuller \\ Department of Earth and Planetary Sciences University of Pittsburgh \\ Pittsburgh, Pennsylvania U.S.A. \\ (Received June 6, 1967)
}

\begin{abstract}
Polycrystalline nickel rods have been subjected to torsional plastic deformation and the dependence of remanence and coercive force observed. The variation of the magnetic properties is interpreted by considering the structural changes induced by deformation. It was found that mechanical hardening or softening due to the deformation is, in general, accompanied by a complimentary magnetic hardening or softening. If the deformation is carried out in the presence of a magnetic field, a component of remanent magnetization is acquired which is dependent upon the magnitude of the field and extent of the deformation The magnetic effects accompanying reverse loading are particularly intimately related to changes in the internal stress state of the rods and provide an explanation for an early self-reversal of magnetization induced in nickel wire by Nagaoka (1888).
\end{abstract}

\section{Introduction}

There is considerable geophysical interest in the stress dependence of the magnetization of rocks and magnetic minerals (Graham, et al, 1957; Stott and Stacey, 1960; Kern, 1961; Nagata and Kinoshita, 1965; Nagata, 1966). The investigations have been primarily concerned with the influence of elastic deformation upon remanence, and interpretations have been based upon bulk magnetostriction effects. Due to the primary concern with the elastic range, little consideration has been given to changes in the internal stress state of the material induced by mechanical (structural) deformation. However, the internal stress, which is determined by the density and configuration of structural defects, is known to affect magnetic properties (Vicena, 1954; Kersten, 1956; Dietrich and Kneller, 1956; Seeger and Kronmüller, 1960). Moreover, the internal stress will itself be affected by externally applied stress, most demonstrably in plastic deformation, but also in the elastic range. The applied stress may create, destroy, or redistribute the defects which define internal stress. As an example of the possible effect of stress, it was suggested by Evison (1962) that plastic flow may have altered the direction of remanence in rocks, although this conclusion for the particular region investigated was refuted by the original field workers (Norris and Black, 1962).

There appears to be a need for an interpretation of irreversible changes in magnetic properties of rocks, such as remanence and coercivity, brought about by applied stress in 
terms of the microscopic deformation processes introduced by the applied stress. As a preliminary step towards this application of materials science to rock magnetism, we have investigated the magnetic effects of plastic deformation in nickel, which is more easily deformed and better understood both mechanically and magnetically than the naturally occurring magnetic minerals. Further, its magnetization is very sensitive to stress. The interpretation of the experimental results has led to an understanding of what may be the first documental self-reversal, i.e., a remanent magnetization aquired in the opposite sense to an external field (Nagaoka, 1888).

\section{Plastic Deformation of Nickel Rods}

\subsection{Introduction}

This experimental study involves the observation and interpretation of changes in remanence and coercive force caused by elastic and plastic deformation of polycrystalline nickel rods in known fields at room temperature. The mode of deformation is torsion, which is convenient because the size and shape of the sample are not changed. The process is, thus, non-destructive mechanically, and demagnetizing effects are invariant. The strain is uniformly distributed along the rod, and cyclic torsion permits readily reproduced experiments. Finally, simple shear, which is the dominant mechanism in all modes of plastic deformation, is produced directly.

\subsection{Experimental Techniques}

Torsion was applied to the samples by using a shop lathe fitted with two chucks which gripped the ends of the rods. The outer fibre shear strain was measured with a calibrated chuck and is reported as angular deflection or percentage strain (circumferential rotation/ deformed length). The field between the chucks is an axial and fairly uniform 1.3 oe. A solenoid was used to null this field and to provide known fields. The measurements of remanence and coercive force were made with a ballistic magnetometer.

\subsection{Dependence of Coercive Force and Remanence upon Deformation}

The magnetic effects of cyclic torsional deformation in the lathe field of 1.3 oe are illustrated in Fig. 1 and 2. Rod A, which is $99.999 \%$ nickel, has a diameter of $0.5 \mathrm{~cm}$ and a length of $7.7 \mathrm{~cm}$ of which $6 \mathrm{~cm}$ is free to twist. The twist of the outer fibre is $160^{\circ}$ giving a strain of $11.7 \%$.

Fig. 1 illustrates the change of coercive force produced by the plastic deformation. The bulk coercivity of the annealed rod is $1.1 \mathrm{oe}$, and increases to 5.8 oe after the ends are severely gripped. It increases, with unidirectional strain, at a decreasing rate to an apparently limiting value of about 24 oe. The behavior in the initial stage of reverse twisting is somewhat surprising. First, there is an increase in coercive force. Then at about $1 \%$ reverse strain, there is a marked decrease. Finally, at $3 \%$ strain a monotonic increase sets in. The effect is more pronounced as cycling is continued.

Fig. 2(a) and (b) illustrate the remanent magnetization acquired by Rod $\mathrm{A}$ in the course of the cyclic deformation. The rod was demagnetized after each measurement for the particular stage of deformation, so that we see the moment acquired between successive 


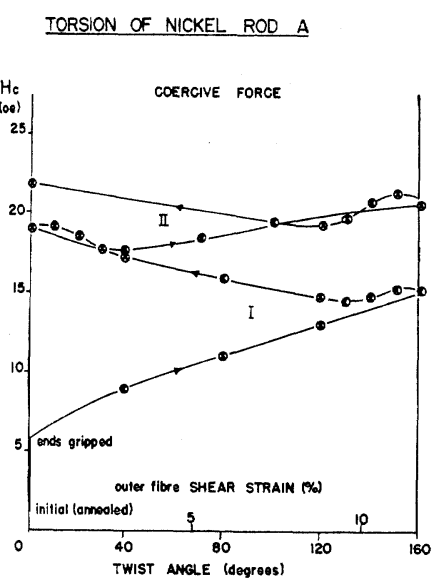

Fig. 1

stages and not the change of a pre-existing moment. Fig. 2(a) shows the total moment developed over a strain increment. The remanence consists of three components. There is an isothermal remanent moment (IRM) induced when the rod is simply placed in the lathe. The IRM acquired in the rod from a state of zero remanence is determined at each experimental step. This can be done because the moment picked up during the previous strain increment is fully demagnetized. The IRM is initially small and decreases to a constant value of about $0.15 \mathrm{emu}$ before the first cycle (Cycle I in the Figure) is complete. This is because the rod has reached a condition of sufficient hardness by the end of the first cycle. There is a pressure or piezo-remanent moment (PRM) developed in the rod ends as a result of the stress exerted by the chuck in gripping the ends. This PRM component was also measured at each step of the deformation history. It reduces to a constant value of about $7 \mathrm{emu}$, after the first cycle of torsion. The third part of the remanent magnetization is what we have called twist remanent (TwRM), which is the additional remanence acquired due to the process of torsional deformation in the presence of a magnetic field. The use of this term is not intended to suggest that a new type of remanence has been identified. Indeed TwRM probably has the same mode of origin as PRM; both magnetizations involve the application of external stress in a magnetic field with shear as the main mode of deformation, although PRM is often used to refer to the changes induced by elastic stress only. Fig. 2(b) indicates the variation of the TwRM with strain, the IRM and PRM having been subtracted. The change in the remanent moment during one incremental stage is indicated by the dotted line in Cycle I.

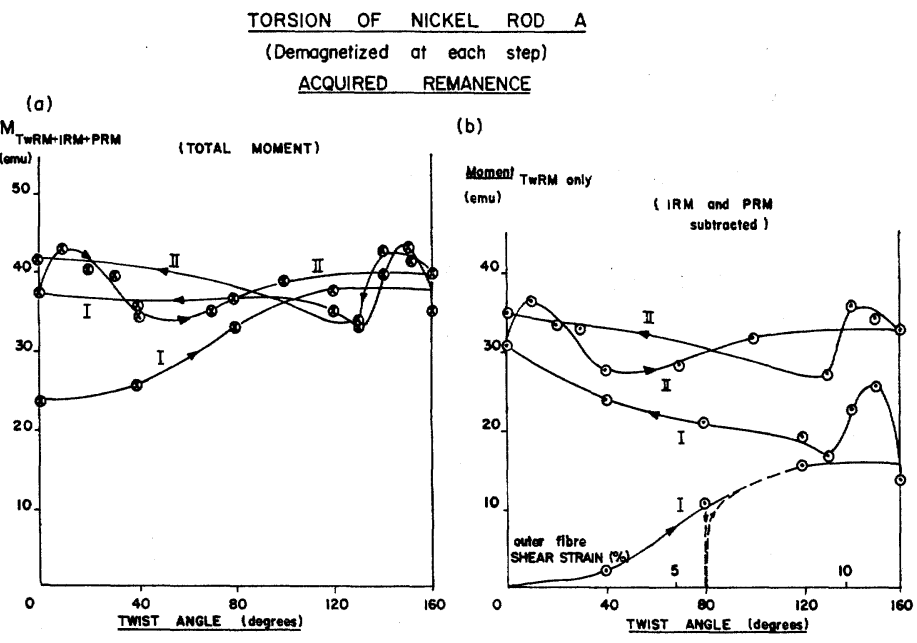

Fig. 2 
We noted from Fig. 1 that in the initial stage of reverse loading the coercive force changed in a surprising manner. Fig. 2(a) shows that the "acquired remanence" follows the pattern of coercive force. In contrast, Fig. 3 shows the results of twisting Rod B $(99.999 \%$ nickel) in the lathe field without demagnetization after each increment of twist. Thus, the deformation operates on a pre-existing stressinduced moment. IRM and PRM are reduced to about 1 emu after one cycle of twist. Reverse twisting (fatigue) of the rod carrying twist remanence causes an initial decrease in the moment. The rod subsequently shows, at a reverse strain of about $3 \%$, the same monotonic increase of moment which it exhibited during the completion of the previous cycle. The limiting value of remanence is approximately $45 \mathrm{emu}$, which is about $30 \%$ of saturation remanence.

\subsection{Mechanical Behavior of the Rods during Cyclic De- formation}

Unidirectional plastic deformation mechanically har-

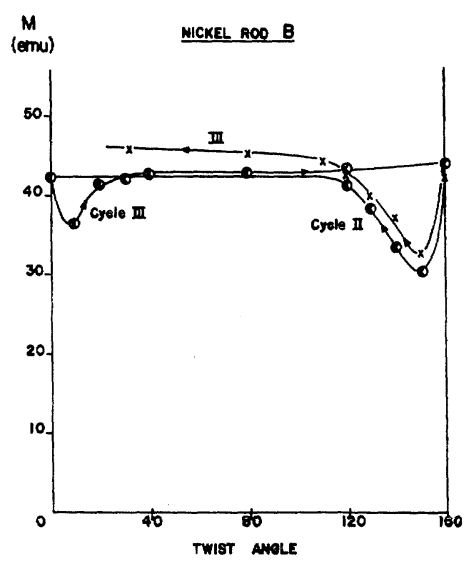

Fig. 3 dens a material. The hardening is due to the increasing density and resulting interaction of defects. Dislocations are created in great numbers by stress approaching the yield point stress, and propagate to give macroscopic slip. They move on slip systems which are suitably oriented with respect to the local stress pattern, piling up where barriers are encountered. The nature of the barriers varies, but atmospheres of point defects, crystal boundaries, large interstitial atoms, or stably intersected or paired dislocations can all impede the motion of mobile dislocations. As the concentration of dislocations increases, it becomes more difficult for new dislocations to be formed, and for those already generated to move any appreciable distance. The material has been "work- or strain-hardened."

When a material is subjected to reverse loading, it exhibits "fatigue softening." This is the "Bauschinger effect," named for its first observer (Bauschinger, 1886; see, for example, Lubahn and Felgar, 1961). The softening is transitory and the strain weakening which gives a lower yield point stress is eventually counteracted by the work hardening accompanying the continued reverse plastic flow. The greater the pre-reversal hardening, the more pronounced is the softening. For cyclic strain, such as in the torsion of the nickel rods, a stage will be reached where the fatigue softening and the normal hardening will combine to give reproducible mechanical behavior for the given strain interval.

The Bauschinger effect is attributed to the relaxation of the internal stress state on reverse loading. The reverse loading disperses dislocation pile-ups on strain hardened slip planes by reverse movement and, in materials with more than one set of slip planes by transfer to other planes more favorably oriented with respect to the principal stresses. Dislocation atmospheres may also be dispersed by transfer or climb of edge dislocations or cross slip of screw dislocations. Moreover, the reverse and transferred movement of dislocations allows the annihilation of pairs of dislocations of opposite sign on the same slip plane. 


\subsection{Interpretation of Dependence of Magnetic Hardness upon Deformation}

Magnetic hardening accompanies mechanical hardening in multidomain materials because the increased density of dislocations impedes not only the movement of disloca. tions, but also the movement of domain walls. The hindrance to wall movement may be due to the magnetostatic effects described by Neel (1946) or to direct interation through the stress fields of the walls and dislocations (for example, Vicena, 1955; Seeger and Kronmüller, 1960). Either mechanism will be most efficient at pinning walls in the vicinity of pile-ups where the defect concentration is locally very high and the internal stress fields reach their maximum value. It is, therefore, surprising to find that reverse loading, which involves the dispersion of pile-ups and mechanical softening, initially produces magnetic hardening as indicated by the increase of the bulk coercive force (see Fig. 1). We interpret this by noting that reverse loading involves the movement of dislocations onto new slip planes into previously unstrained regions, so that walls which were unimpeded now interact with these dislocations and wall motion is further inhibited. However, fatigue does involve the dispersion of the pile-ups and, therefore, must cause a decrease in the size of the regions of stress concentration, and of the maximum stress values present. It is clear from our results that the magnetic "softening" dominates between $1 \%$ and $3 \%$ reverse strain, which is the range in which the coercive force experiences a pronounced drop. We interpret this as due to the continued reduction in size of the regions of stress concentration and annihilation of some structural defects with effective intersection of new and old slip systems. After $3 \%$ reverse strain is exceeded, the plastic flow associated with the new unidirectional deformation dominates to give magnetic hardening with the reconstitution of dislocation pile-ups on slip planes.

The interpretation of the magnetic behaviour which has been given for deformation of nickel can be used to predict behaviour in other materials, and the validity of the model can be tested. For example, the model predicts certain differences of behaviour dependent upon the crystal structure of the material. If the material has only one slip plane, then the initial increase of coercive force observed in the fatigue of nickel should be much less significant because the dislocations are confined to a single set of planes and cannot move into undeformed regions as readily as they do in nickel. Moreover, the decrease in coercive force should continue over a larger strain interval because complete softening on the unique set of slip planes must occur prior to renewed strain hardening. In contrast, if there are many sets of slip planes, as there are in nickel, slip and hardening on new planes occurs with the original slip plane system in a state of residual hardening. The observed dependence of the coercive force of the hexagonal form of cobalt, which has one slip plane, upon reverse loading is consistent with the predictions of our model.

\subsection{Interpretation of Dependence of Remanence upon Deformation}

It has been demonstrated that domain configurations in a variety of materials can be totally changed by external stress; e.g., in ferromagnetic nickel (Kirenskii, et al, 1964) and silicon-iron (Dijkstra and Martius, 1953), and in ferrimagnetic magnetite (Bogdanov and Vlasov, 1966). The overall pattern, and, thus, direction and magnitude of magnetization be- 
comes controlled by magnetoelastic energy $\left(E_{\lambda \sigma}\right)$ for sufficient stress, rather than by magnetocrystalline anisotropy energy $\left(E_{k}\right)$. Moreover, there is a magnetoelastic hysteresis.

An approximate determination of the stress required to produce a stress-controlled domain configuration may be made by equating the magnetoelastic and magnetocrystalline energies. We may take as a criterion the energy required to deflect the direction of magnetization from one "easy" direction to another. Consider the energy anisotropy involved in deviating a moment from a [111] easy direction to a [11] ] direction over the [110] magnetocrystalline energy barrier, for an applied stress along the [111] direction, in moderately worked nickel.

$$
\begin{aligned}
\left|\Delta E_{\lambda \sigma}\right| & =\left|E_{\lambda \sigma[111]}-E_{\lambda \sigma[110]}\right| \\
& =12.5 \times 10^{-6} \sigma \mathrm{ergs} / \mathrm{cm}^{3}
\end{aligned}
$$

with stress $\sigma$ in dynes $/ \mathrm{cm}^{2}$, and

$$
\begin{aligned}
\left|\Delta E_{k}\right| & =\left|E_{k[111]}-E_{k[110]}\right| \\
& =0.27 \times 10^{4} \mathrm{ergs} / \mathrm{cm}^{3}
\end{aligned}
$$

$\left|\Delta E_{k}\right|$ is the energy barrier which the magnetoelastic anisotropy must overcome, in order to control the pattern of magnetization. It may be shown that for the case outlined, a factor of $3 / 2$ applied to the stress obtained by direct equation of the two energies reduces the barrier created by the magnetocrystalline energy to essentially zero. Hence, for $E_{\lambda \sigma}$ to dominate over $E_{k}$,

$$
\begin{aligned}
\left|\Delta E_{\lambda \sigma}\right| \geq 3 / 2\left|\Delta E_{k}\right| & \\
12.5 \times 10^{-6} \sigma & \geq 3 / 2 \times 0.27 \times 10^{4} \\
\sigma & \geq 3.24 \times 10^{8} \text { dynes } / \mathrm{cm}^{2}
\end{aligned}
$$

The stress must, thus,exceed about $330 \mathrm{kgms} / \mathrm{cm}^{2}$. This is in accord with the experimental observations of Kirenskii, et al, (1964). This stress is considerably less than the estimated "true" yield point stress in shear for moderately worked nickel of about $2200 \mathrm{kgms} / \mathrm{cm}^{2}$, which is exceeded in plastic deformation.

Thus, during deformation in our experiments, the magnetization is controlled (except in that small central core of the rod in low elastic stress) by magnetoelastic energy, in conjunction with magnetostatic energy due to the external field. The irreversible magnetic effect, or acquired remanent moment, arises because, after the external stress is removed, the magnetoelastic energy barriers created by the remanent strain pin part of the magnetization. Domain walls are constrained, and magnetization relaxation inhibited.

The interpretation of the detailed dependence of remanence upon fatigue loading follows from the above discussion. When the remanence is investigated by the incremental technique (Fig. 2(a) and (b)), the behaviour of remanence follows that of the coercive force. In contrast, if we observe the effect on a previously acquired strain-induced moment, there is an initial decrease of remanence followed by recovery (see Fig. 3). This is interpreted as due to unpinning of remanence (i.e., increased mobility of domain walls) because of the dispersion of pile-ups and consequent decrease in the size of the regions of stress concentration. The loss of remanence due to this effect is eventually compensated by 
the increase in number and size of pile-ups brought about by the new unidirectional deformation.

The magnetomechanical model for the dependence of remanence upon internal structural state can be tested in the same way as was the model for coercive force. The reduction of pre-existing strain-induced moments due to fatiguing should be much more pronounced in a material with a single slip plane. This is because a single slip plane must be completely softened before the hardening due to reverse loading again dominates. Ex perimental results for hexagonal cobalt are consistent with this prediction. As mentioned, in nickel other slip planes can be activated and worked before the total softening of previously worked planes, and the proportional decrease in remanence is less.

\section{The Self-Reversal of Magnetization Observed by Nagaoka}

\subsection{Introduction}

A remarkable self-reversal of magnetization was induced in nickel wire by Nagaoka (1888 a and b, $1889 \mathrm{a}$ and b). A wire could be subjected to cyclic torsional deformation in a magnetic field unitil a reproducible and symmetric pattern of the dependence of the magnetization upon torsion was achieved. If the wire was then progressively axially loaded during the torsional fatiguing, for sufficient tension a magnetization directed opposite to the field was developed during the half cycle of reverse twist. This self-reversal could be suppressed for a given axial load if a field of sufficient magnitude was applied. Although confirmed in the laboratories of Kelvin and Weiss, no explanation for this phenomenon was advanced, presumably because the necessary magnetomechanical correlation of structural state and magnetic response was not available.

\subsection{Experimentation}

Nagaoka used polycrystalline nickel wire $40 \mathrm{~cm}$ long and $1 \mathrm{~mm}$ in diameter. The known impurities were $1.7 \%$ iron and some carbon. The initial mechanical state of the wire in a particular experiment can only be inferred from the reported magnetic properties because a single wire would be used for several experiments involving successive work treatments. The magnetization of the wire was measured with a suspended fibre magnetometer. The moments measured were those parallel to the wire axis, thus, in the direction of the external fields applied by a solenoid.

\subsection{Magnetic Behaviour of Deformed Nickel Wire}

In the initial state the wire which Nagaoka used had a saturation moment of about 360 $\mathrm{emu} / \mathrm{cm}^{3}$ and a coercive force of $10 \mathrm{oe}$. After a preliminary torsional outer fibre shear strain of $3^{\circ}$ twist $/ \mathrm{cm}(0.26 \%)$, the saturation remanent moment increased by about $50 \%$. When the wire was subjected to axial tension, the axial susceptibility and remanence were reduced; for a tensile stress of $1270 \mathrm{kgms} / \mathrm{cm}^{2}$ remanence decreased to about $14 \%$ of the value in the unstressed state. If the wire was subjected to torsion and tension simultaneously, the axial susceptibility and the remanence were larger than for pure tension, but remained smaller than the unstressed values.

The hysteretic magnetic behaviour of the wire during reverse loading was found to 
vary depending upon the amount of initial strain. For a small twist of, say, $\pm 1.7^{\circ} / \mathrm{cm}$. (outer fibre shear strain of $\pm 0.15 \%$ ), the return curve of magnetization against strain was above the outward curve (Nagaoka, 1889b) giving "positive hysteresis". In contrast, if a larger strain of, say, $\pm 4.5^{\circ} / \mathrm{cm}( \pm 0.49 \%)$ was used, the return curve fell below the outward curve (negative hysteresis).

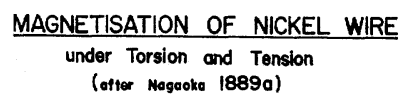

(a) Tensile Load $\underline{\left(\mathrm{kgms} / \mathrm{cm}^{2}\right)}$
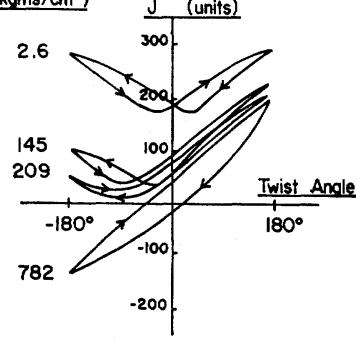

$H_{\text {ex }}=2.47 \mathrm{oe}$

(b)

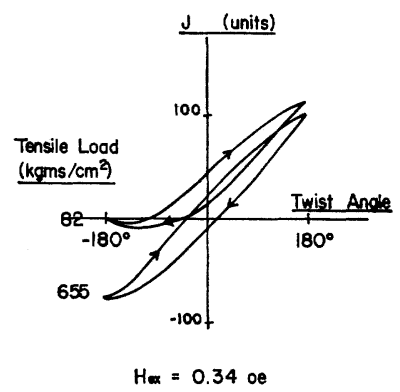

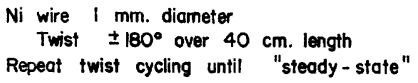

Fig. 4

It was found that repeated twisting of about seven cycles was required to produce a "steady state" or reproducible magnetic behaviour for the strain range of $\pm 0.39 \%$. Typical results are shown in Fig. 4(a). As the tensile load increased, the moment decreased, and the curve of magnetization against torsional deformation lost its symmetry. In an external field of $2.47 \mathrm{oe}$, a tensile stress of $145 \mathrm{kgms} / \mathrm{cm}^{2}$ displaces the nodal point and reduces the moment acquired during the reverse twist. A stress in excess of about $225 \mathrm{kgms} / \mathrm{cm}^{2}$ is sufficient to reverse the magnetization during the reverse loading. In a smaller external field of $0.34 \mathrm{oe}$, a stress greater than about $30 \mathrm{kgms} / \mathrm{cm}^{2}$ reversed the magnetization during the reverse twist (Fig. $4(\mathrm{~b})$ ).

\subsection{Interpretation of Nagaoka's Results}

The results obtained by Nagaoka can be understood in the light of the experiments and interpretation given in Section 2. The wire which he used was evidently in a state of intermediate strain hardening, judging by its coercive force of 10 oe. After the preliminary torsional deformation its internal stress was substantially increased. The stress was elastic throughout the cross-section; the maximum strain is less than the estimated "true" yield point strain in shear for moderately worked nickel of about $0.3 \%$. The relaxation of saturation magnetization was inhibited by the new barriers to wall movement and rotation, and the saturation remanence increased. During elastic tension an "easy" plane of magnetizaiton is defined perpendicular to the axis of tension, because of the negative magnetostriction. It is the $\max / \mathrm{min}$. plane of the strain ellipsoid (see Footnote*), as the configuration of principal stresses is $\sigma_{1}>\sigma_{2}=\sigma_{3}$ where $\sigma_{1}$ is the applied tensile stress. Axial remanence is 
reduced because the majority of domains are now oriented perpendicular to the wire axis. Within the favoured plane defined by the tension in the wire, domain moments are preferentially aligned circumferentially because of the demagnetizing effect.

For simple torsion, the preferred easy direction is again parallel to the least strain axis; and is, thus, initially at an angle of approximately $45^{\circ}$ to the wire axis and parallel to the plane of the surface. This is because the intermediate principal stress $\sigma_{2}$, which is in the direction of the local normal to the surface, is not equal to the least stress as it was in simple tension. The stress ordering is $\sigma_{1}>\sigma_{2}>\sigma_{3}$ where $\sigma_{2}=\frac{\sigma_{1}+\sigma_{3}}{2}$ for initial torsion. $\sigma_{1}$ is the component of applied shear stress resolved along the greatest strain-axis of the strain ellipsoid. As the torsional deformation proceeds, the effective strain ellipsoid rotates and the preferred direction of magnetization rotates toward the wire axis. The combination of tension with torsion will bring $\sigma_{2}$ closer to $\sigma_{3}$ in magnitude. It should be pointed out that in plastic deformation the strain ellipsoid, which is a representation for elastic deformation of a homogeneous isotropic material, will not retain its integrity on a microscopic scale. There will be shear slip, predominantly on the fault system aligned approximately normal to the wire axis for initial torsion, but which will rotate with continuing deformation. It is still possible, however, to define a macroscopic "effective" strain ellipsoid.

The magnetic behaviour during reverse loading depends upon the influence of Bauschinger softening. If it is pronounced, then there is a marked magnetization relaxation, and the return curve of magnetization against strain falls below the outward curve. If the material is unworked or has suffered only small strain, there is minimal strain hardening; and thus, negligible Bauschinger softening. The magnetization relaxation is, thus, further inhibited by the reverse straining and the magnetization-strain curve shows positive hysteresis.

The curves of magnetization against strain shown in Fig. 4 are a reflection of the magnetic response to a steady state in the balance of strain hardening and Bauschinger softening achieved after repeated cycling over a particular strain range. The total strain for unidirectional working is $0.78 \%$, which is estimated to be in the range of moderate shear deformation. The uppermost curve in Fig. 4(a) is symmetrical because the fatigue softening in the first reverse loading (cf. Fig. 3) is exactly the amount required to ensure that the reverse strain hardening recovers the initial moment at "zero twist". The symmetry is destroyed if the same strain is produced when the wire is subjected to greater tension. This is because the same strain involves greater working when the wire is under

* Footnote

The "strain ellipsoid" to which we refer is a deformation surface produced by elastic strain of a unit sphere. If the equation of the sphere is $x^{2}+y^{2}+z^{2}=1$ and the values of the principal strains are $\epsilon_{1}, \epsilon_{2}$, and $\epsilon_{3}$, then $x^{\prime}, y^{\prime}$, and $z^{\prime}$ which define the strain ellipsoid are of the form $x^{\prime}=(1+\epsilon) x$. The equation of the strain ellipsoid is thus

$$
\frac{x^{\prime 2}}{\left(1+\epsilon_{1}\right)^{2}} \frac{y^{\prime 2}}{\left(1+\epsilon_{2}\right)^{2}} \frac{z^{\prime 2}}{\left(1+\epsilon_{3}\right)^{2}}=1
$$


(a)

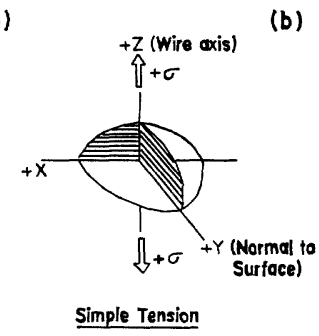

(b)

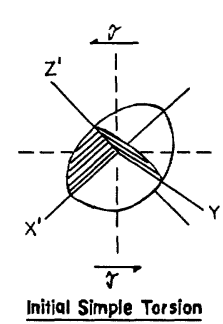

(c)

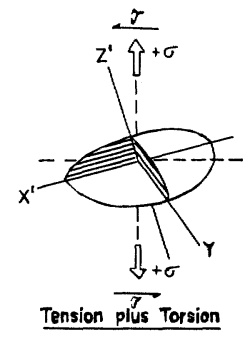

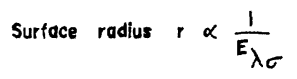

Fig. 5

greater tension; the axial tension tends to oppose the deforming effect of torsion, and further elongates the strain ellipsoid. This, in turn, enhances Bauschinger softening because the pre-strained material is in a more highly worked state. The reverse hardening sets in later, and the "nodal point" is displaced towards "negative" twist (e.g., Fig 4(a), for 145 $\mathrm{kgms} / \mathrm{cm}^{2}$ ). The moment also decreases due to the effect of the elastic tension, as was explained previously.

The definition of the preferred orientation of magnetization by applied stress may be illustrated by use of a representation surface. Fig. 5 shows the energy relationships effective in controlling the domain configuration, and, the observed magnetization. It shows the proposed explanation for the stress-induced self-reversal. If a volume element is subjected to simple tension the strain ellipsoid elongates in the direction of greatest stress. With sufficient stress to allow stress control of magnetization to dominate other energies such as magnetocrystalline anisotropy, an ellipsoidal surface inverse to the strain ellipsoid may be defined with a radius vector inversely proportional to the magnetoelastic energy. This is applicable for a material with negative magnetostriction, such as nickel. The surface is shown in Fig. 5(a), using two octants of the three-dimensional surface, with the $y$ axis directed normal to the wire surface. The ellipsoid is proportional, in a first approximation, to the magnetization because the majority of domains will lie in the direction of least energy. For Fig. 5(a), this is the $x y$ plane. The assumption of strong stress control of magnetization is acceptable for worked nickel because at a stress beyond the initial part of the elastic range, magnetoelastic energy exceeds the low anisotropic crystalline energy characteristic of a cubic material.

When the wire volume element is subjected to simple shear by torsion, the "magnetoelastic ellipsoid" rotates in the $x z$ plane (Fig. 5(b)). The magnetization is preferentially parallel to the least strain $x^{\prime}$ axis, about $45^{\circ}$ from the wire axis $z$. The ellipsoid is triaxial because of the principal stress relationships. Fig. 5(c) shows the effect of superposing an axial tension on the torsion. The "equatorial" $x^{\prime} y$ plane is more favourable for domain magnetization directions, with the $x^{\prime}$ direction being preferred within the plane. 
It should be noted that for a volume element in the wire, particularly near the surface, the effective magnetization ellipsoid is further distorted from the magnetoelastic constraint by the influence of the demagnetizing energy. This will give a relative "shortening" of the $y$ axis so that the $x$ axis (Fig. 5(a)) or $x^{\prime}$ axis (Fig 5(b) and (c)) is preferred energetically.

Consider now the total energy anisotropy affecting magnetization, in the presence of an external field. Fig. 5(d), (e), and (f) portrays a surface with radius vector inversely proportional to the sum of the dominant energies in a wire subjected to tension and torsion. For sufficient stress to overbalance the influence of magnetocrystalline anisotropy, these energies are the magnetoelastic $\left(E_{\lambda \sigma}\right)$, magnetostatic $\left(E_{H}\right)$ due to the applied field, and demagnetizing $\left(E_{N J}\right)$.

\section{MODEL FOR MAGNETIZATION REVERSAL}

(d)

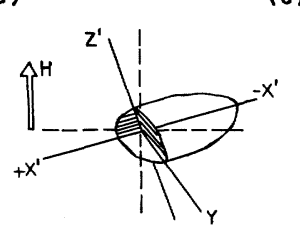

(e)

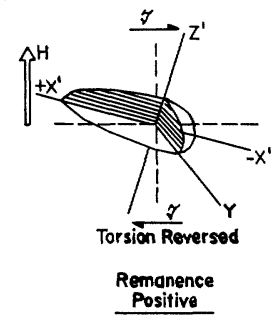

Remanence
Positive

Positive

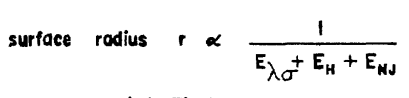

(f)
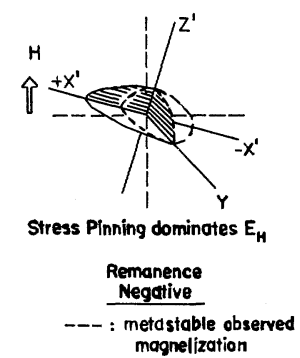

opplied Field $=H$

Fig. 5

In an external field, the moments are constrained to lie in the $x^{\prime}$ direction by the magnetoelastic energy, but the magnetostatic energy is less for moments oriented in the $-x^{\prime}$ direction than in the $+x^{\prime}$ direction (Fig. 5(d). The magnetization is predominantly in the $-x^{\prime}$ sense, and moment is "positive" (i.e., in the direction of the field). When the direction of twist is reversed, the effective strain ellipsoid rotates through the horizon plane and the magnetization ellipsoid shifts to the new configuration $x^{\prime} y z^{\prime}$, seen in Fig. 5(e). If the preference for $+x$ or $-x$ direction is determined only by magnetostatic energy, then $+x$ is now the preferred direction for moment vectors because it is in the direction of the applied field. The moment would still be positive. However, another possibility arises. We have assumed previously that the minimum energy configuration of domains is always achieved. This need not happen; a metastable configuration can occur which could give the self-reversal. When the reverse twist is applied, some stress-pinned domains will rotate with the axis of least energy. These domains will nor reverse poldrity, so that the bulk moment could still be in the $-x^{\prime}$ direction. This direction now lies below the horizon plane, giving a "negative" axial moment. Domain walls are effectively restrained from free movement by stress barriers created by the stress state of the material and the domain configuration is pinned by stress. Thus, it is not free to alter substantially to satisfy the 
energy minimum preferred by magnetostatic energy. This case of metastable equilibrium is illustrated in Fig. 5(f). As the axial tension is increased the wire becomes more strongly stressed, because more torsional shear stress must be applied to attain the same strain. For a given external field, the reversal, thus, occurs for a tensile stress great enough to "pin" sufficient domain walls. For a sufficiently large external field the walls may migrate in response to the magnetostatic energy and the bulk moment will not be pinned in the negative direction by residual stress.

This model depends on the relative magnitude of magnetoelastic energy available for pinning moments, which is linearly proportional to stress, and the magnetostatic energy, which is linearly proportional to applied field. The relation between the "critical" stress to induce a reversal, and field, should, therefore, be linear. This was observed: For $\pm 4.5^{\circ} /$ $\mathrm{cm}$ twist the reversal stress was about $85 \mathrm{kgms} . \mathrm{cm}^{-2} /$ oe (adapted from Nagaoka, 1888a). With this experimental observation we can equate the magnetostatic and magnetoelastic energies at the critical point for reversal at a field of 1 oe. Using the value of $J_{S}$ indicated by Nagaoka's work, and a direction of $J_{\mathcal{S}}$ of about $45^{\circ}$ on the wire axis as favoured by torsion, the energies are:

$$
\begin{aligned}
E_{H} & =J_{S} \mathrm{H} \cos \theta \\
& \approx 360 \times 1 \times(1 / \sqrt{2}) \\
& =255 \mathrm{ergs} / \mathrm{cm}^{3} \\
E_{\lambda \sigma} & =-3 / 2 \lambda_{S} \sigma \cos ^{2} \theta \text { for polycrystalline material } \\
& \approx-3 / 2\left(-41 \times 10^{-6}\right) \times 85 \times 980,000 \times(1 / \sqrt{2})^{2} \\
& =256 \mathrm{ergs} / \mathrm{cm}^{3}
\end{aligned}
$$

Thus, at this critical point $E_{H} \approx E_{\lambda \sigma}$ and there is sufficient magnetostatic energy to overcome the magnetoelastic energy in enough of the bulk material to suppress the reversal phenemenon. For a given field, sufficient stress (i.e., applied tensile load) will induce a reversal, and equivalently for a given stress a great enough field will prevent the reversal. The model is dependent on a negative magnetostriction, and predicts that a reversal would not occur for a material with positive magnetostriction under tension. The absence of the phenomena in iron is consistent with this prediction.

\subsection{Geophysical Significance}

Our work to date on the magnetic response of the mineral magnetite to applied elastic stress indicates a behaviour similar to that of nickel. While acknowledging differences between the ferrite magnetite and the metal nickel, an analogy can be drawn the two because of some striking similarities in mechanical and magnetic behaviour. It has been demonstrated both experimentally and theoretically, that magnetite is as responsive to stress as nickel to within a factor of two. It is suggested that certain aspects of irreversibility of remanence as a function of stress may be understood using mechanical considerations such as hysteresis of structural changes. It is hoped that our understanding of the effect of plastic deformation on remanence and coercivity in nickel, may be extended to magnetite in the deformation environment of the Earth's upper crust. It may be of interest to note that for magnetite, which has positive magnetostriction, the combination of compression, 
such as overburden pressure, and fatigue shear-flow might produce a self-reversal.

\section{Ackowledgment}

This work has been financed by the U.S. National Science Foundation, Grant Number, GP 5546. The first named co-author is indebted to the Andrew W. Mellon Educational and Charitable Trust for fellowship support. Thanks are due Professor R.D. Wyckoff for use of shop facilities at the University.

\section{References}

Bauschinger, J., Mitt. mech. tech. Lab. München (Munich) 1886 ; Dinglers Polytech. J., 226, 1886.

Bogdanov, A.A., and A.Ya. Vlasov, Adad. Nauk SSSR, Izv. Fizika Zemli (trans., Physics of the Solid Earth), 1, 42, 1966.

Dietrich, H., and E. Kneller, Zeit. fur Metallkunde, 47, 716, 1956.

Dijkstra, L., and U. Martius, Revs. of Mod. Phys., 25, 146, 1953.

Evison, F.F., Nature, 194, 644, 1962.

Graham, J.W., A.F. Buddington, and J.R. Balsley, J. Geophys. Res., 62, 465, 1957.

Kern, J.W., J. Geophys. Res., 66, 3807, 1961.

Kersten, M., Z. angew. Physik, 80, 496, 1956.

Kirenskii, L.V., M.K. Savchenko, I.F. Degtyarev, S.V. Kan, I.P. Antipin, Y.D. Tropin, and I.S. Edel'man, Akad. Nauk SSSR Bulletin, Physical Series, 28, Part I, 465, 1964.

Lubahn, J.D., and R.P. Felgar, Plasticity and Creep of Metals, J. Wiley and Sons, 1961.

Nagaoka, H., J. College of Science, Tokyo (Imperial) Univ., 2, 283, 1888a.

Nagaoka, H., J. College of Science, Tokyo (Imperial) Univ., 2, 304 1888b.

Nagaoka, H., Phil. Mag., Series 5, 27, 117, 1889a, plus addendum note by J. T. Bottomley and A. Tanakadate, p. 133.

Nagaoka, H., J. College of Science, Tokyo (Imperial) Univ., 3, 189, 1889b.

Nagata, T., J. Geomag. and Geoelect., 18, 81, 1966.

Nagata, T., and H. Kinoshita, J. Geomag. and Geoelect., 17, 121, 1965.

Neel L., Ann. de l'Universite de Grenoble, 22, 299, 1946.

Norris, D.K., and R.F. Black, Nature, 194, 646, 1962.

Seeger, A., and H. Kronmüller, J. Phys. Chem. Solids, 12, 298, 1960.

Stott, P.M., and F.D. Stacey, J. Geophys. Res., 65, 2419, 1960.

Vicena, F., Czech. J. Phys., 4, 419, 1954.

Vicena, F., Czech. J. Phys., 5, 480, 1955. 